\title{
Recurrent Duplication and Diversification of Acrosomal Fertilization Proteins in Abalone
}

\author{
J. A. Carlisle ${ }^{1 *}$, M. A. Glenski² ${ }^{2}$ W. J. Swanson ${ }^{1}$ \\ 1. University of Washington Medical School, Genome Sciences Department \\ 2. Gonzaga University
}

${ }^{*}$ Corresponding author - J. A. Carlisle (jcarlisl@uw.edu)

\begin{abstract}
Reproductive proteins mediating fertilization commonly exhibit rapid sequence diversification driven by positive selection. This pattern has been observed among nearly all taxonomic groups, including mammals, invertebrates, and plants, and is remarkable given the essential nature of the molecular interactions mediating fertilization. Gene duplication is another iimportant mechanism that facilitates the generation of molecular novelty. Following duplication, paralogs may parse ancestral gene function (subfunctionalization) or acquire new roles (neofunctionalization). However, the contributions of duplication followed by sequence diversification to the molecular diversity of gamete recognition genes has been understudied in many models of fertilization. The marine gastropod mollusk abalone is a classic model for fertilization. Its two acrosomal proteins (lysin and sp18) are ancient gene duplicates with unique gamete recognition functions. Through detailed genomic and bioinformatic analyses we show how duplication events followed by sequence diversification has played an ongoing role in the evolution of abalone acrosomal proteins. The common ancestor of abalone had four members of its acrosomal protein family in a tandem gene array that repeatedly experienced positive selection. We find that both sp18 paralogs contain positively selected sites located in different regions of the paralogs, consistent with a subfunctionalization model where selection acted upon distinct binding interfaces in each paralog. Further, a more recent species-specific duplication of both lysin and sp18 in the European abalone H. tuberculata is described. Despite clade-specific acrosomal protein paralogs, there are no concomitant duplications of egg coat proteins in $H$. tuberculata, indicating that duplication of egg proteins per se is not responsible for retention of duplicated acrosomal proteins. We hypothesize that, in a manner analogous to host/pathogen evolution, sperm proteins are selected for increased diversity through extensive sequence divergence and recurrent duplication driven by conflict mechanisms.
\end{abstract}

\section{Introduction}

Despite their essential role in many organisms, genes functioning in fertilization or sexual reproduction are often rapidly diverging between closely related species including mammals, birds, fish, and invertebrates (Carlisle \& Swanson, 2020; Swanson, Nielsen, \& Yang, 2003; Swanson \& Vacquier, 2002). Some pairs of interacting sperm and egg gamete recognition proteins have been shown to be rapidly co-evolving, pointing to sexual conflict or sexual selection driving the rapid evolution of fertilization genes (Bianchi, Doe, Goulding, \& Wright, 2014; Clark et al., 2009; Grayson, 2015; Kamei \& Glabe, 2003). This rapid diversification of 
sperm and egg gamete recognition proteins at a sequence level can result in species-specific fertilization function (Avella, Baibakov, \& Dean, 2014; Raj et al., 2017; Zigler, McCartney, Levitan, \& Lessios, 2005). Investigations into the evolution of reproductive genes paired with characterization of species-specific function can provide unique insights into infertility and reproductive isolation (Lehmann, 2018). In addition to sequence evolution, gene duplication can also contribute to the molecular diversification of reproductive protein families. Drosophila seminal fluid proteins often undergo duplication and diversification, with many of these duplications being species-specific (Almeida \& Desalle, 2009; Doty, Wilburn, Bowen, Feldhoff, \& Feldhoff, 2016; Findlay, Yi, Maccoss, \& Swanson, 2008; Sirot et al., 2014; Wagstaff \& Begun, 2005; D. B. Wilburn, Arnold, J. A., Houck, L. D., Feldhoff, P. W., Feldhoff, R. C., 2017). Mammalian genes mediating fertilization including CatSper, Izumo, and the ZP gene families also demonstrate interesting patterns of duplication (Aagaard, Vacquier, MacCoss, \& Swanson, 2010; Cai \& Clapham, 2008; Cooper \& Phadnis, 2017; Grayson \& Civetta, 2012). Here, we investigate how both sequence diversification and duplication together contribute to the molecular diversification of fertilization proteins.

The marine gastropod abalone (Genus Haliotis) is a classic model system for studying the function of gamete recognition proteins and their evolution. Abalone sperm have an extremely large acrosome containing two gamete recognition proteins, lysin and sp18 (Lewis, Leighton, \& Vacquier, 1980). Lysin is the sperm mediator of the dissolution of the egg's vitelline envelope (VE), and sp18 is thought to mediate sperm-egg plasma membrane fusion (Carlisle \& Swanson, 2020; D. B. Wilburn, Tuttle, Klevit, \& Swanson, 2018). The abalone egg VE is an elevated glycoproteinaceous layer homologous to the mammalian egg zona pellucida (ZP) (Carlisle \& Swanson, 2020). The abalone VE and mammalian ZP are biochemically and structurally similar (Mozingo, Vacquier, \& Chandler, 1995) and both contain proteins with ZP-N domains (Avella et al., 2014; Carlisle \& Swanson, 2020). Binding between lysin and the ZP-N domains of the vitelline envelope receptor for lysin (VERL) leads to the non-enzymatic dissolution of the VE (Aagaard, Springer, Soelberg, \& Swanson, 2013; Raj et al., 2017; Swanson \& Vacquier, 1997). Three-dimensional structures of lysin and VERL have been investigated using NMR and crystallography and their ability to bind each other have been quantified (Aagaard et al., 2013; Kresge, Vacquier, \& Stout, 2000, 2001; Raj et al., 2017; D. B. Wilburn et al., 2018). The highly fusogenic protein sp18 is the putative mediator of sperm-egg plasma membrane fusion in abalone (Kresge et al., 2001; Swanson \& Vacquier, 1995). The structure of sp18 has been determined via crystallography, but its binding receptor of is unknown (Kresge et al., 2001).

Investigations into the evolution of abalone reproductive proteins have provided valuable insights within the field of reproductive biology. Lysin, sp18 and VERL have each been shown to evolve under positive selection by analysis of the ratio of rates of nonsynonymous substitutions to rates of synonymous substitutions $\left(d_{N} / d_{s}>1\right)$ (Galindo, Vacquier, \& Swanson, 2003; Lee, Ota, \& Vacquier, 1995; Swanson \& Vacquier, 1995). Further, population genetic analysis indicate that lysin and its binding partner VERL are coevolving with each other (Clark et al., 2009). Previous studies of abalone egg and sperm gamete recognition proteins hint at the importance of duplication events for their evolution. Despite their divergent functions in reproduction and low sequence similarity, sp18 and lysin are paralogs with similar three-dimensional protein structures (Kresge et al., 2001). The common ancestor of lysin and sp18 is hypothesized to have mediated both VE dissolution and sperm egg fusion, then parsed these combined ancestral functions post-duplication to lysin and sp18, respectively.

Additional evidence suggests that duplication may be contributing to the evolution of abalone sperm fertilization proteins on a more recent timescale. The European abalone $H$. tuberculata 
has a species-specific duplication of lysin (Clark, Findlay, Yi, MacCoss, \& Swanson, 2007). On the egg side there is also evidence of extensive gene duplication. In addition to VERL, abalone VEs contain $\sim 30$ homologous VEZP proteins containing ZP-N domains (Aagaard et al., 2010; Aagaard, Yi, MacCoss, \& Swanson, 2006). Many of these VEZPs may be structural components of the VE that play no role in gamete recognition (Killingbeck \& Swanson, 2018). However, one protein (VEZP-14) is a close paralog of VERL that has undergone positive selection and is capable of binding lysin (Aagaard et al., 2013). Abalone VEZPs have only been described in two species ( $H$. rufescens, and $H$. fulgens) from the North American clade. It is unknown whether there is variation in gene content across more distantly related abalone species (Aagaard et al., 2006).

In this study we investigated the contributions of duplication and sequence diversification to the evolution of proteins mediating fertilization across the genus Haliotis. Using new testes and ovary transcriptomic data and published genome assemblies we discovered novel duplications of the acrosomal proteins lysin and sp18. Some of these paralogs are ancestral to abalone and others are clade-specific. Further we discover signatures of positive selection in many of the paralogs and identify patterns of positively selected sites indicative of subfunctionalization. Our detailed evolutionary genomic analysis reveals how recurrent patterns of duplication paired with diversification led to the evolution of abalone gamete recognition proteins and their variation between species. Repeated duplications within the protein family containing lysin and sp18 parallels the duplication and diversification of other reproductive protein families, such as mammalian Izumo and Catsper families.

\section{Materials and Methods}

\section{PacBio Library Preparation and Sequencing}

To identify potential transcripts present in abalone gonadal tissue, methods were adapted from the PacBio Iso-seq protocolto create cDNA libraries. Ovary and testes transcriptome libraries were prepared for PacBio sequencing. RNA was extracted from $H$. tuberculata ovary and testes samples by cesium chloride density gradient centrifugation (MacDonald, Swift, Przybyla, \& Chirgwin, 1987). RNA samples were enriched for mRNA by using the Oligotext mRNA Mini Kit from Qiagen. Purified mRNA was used as the template for single stranded cDNA synthesis using the Clontech SMARTer cDNA Synthesis Kit. The cDNA was amplified by PCR using the AccuPrime High-Fidelity Taq system (Invitrogen, Carlsbad, CA, USA). Double stranded synthesis conditions were optimized with the following PCR program: ${ }^{\circ} \mathrm{C}$ for 2 minutes, followed by 20 cycles of $94^{\circ} \mathrm{C}$ for $30 \mathrm{~s}, 55^{\circ} \mathrm{C}$ for $30 \mathrm{~s}$, and $68^{\circ} \mathrm{C}$ for 10 minutes. We used unique identifying barcoded PCR primers to amplify testes (barcode: CTGCGTGCTCTACGAC) and ovary (barcode: TCAGACGATGCGTCAT) cDNA. Because of size bias during PacBio sequencing, double stranded cDNA was fractionated using Ampure XP Beads (Beckman Coulter Life Sciences) into two fractions (Ratio of $4: 1$ of $0.45 x: 0.6 x$ size selection). Testis and ovary cDNA were sequenced using the PacBio RSII and was performed by the Washington State University Genomics Core.

\section{Identification of Acrosomal Protein Paralogs}

Sequences of sp18 and lysin from the genus Haliotis were retrieved from NCBI Genbank sequence repository (accession numbers for lysin: L26270-79, L26281-83, L35180-81, L36589, M34388-89, M59968-72, M98874-75, HM582239; accession numbers for sp18: L36552-54, L36589-90, MN102340-42). These sp18 and lysin sequences were used as the initial query 
sequences when identifying paralogs in abalone transcriptomes and genomes. Queries of the $H$. rufescens Illumina-based testis transcriptome (Palmer et al., 2013) and the $H$. tuberculata PacBio testes transcriptome were conducted with tblastn with an e-value cutoff of $1 \mathrm{e}-10$. Significant matches from the testes transcriptomes were searched against the NCBI sequence repository (July 2020) using tblastn in order to confirm homology to lysin or sp18 (McGinnis \& Madden, 2004). New sequences were uploaded to Genbank under accession numbers OK491874-OK491877.

Regions of publicly available abalone genomes containing novel acrosomal protein duplications of sp18 and lysin were identified by using tblastn with a e-value cutoff of 1e-10 (Botwright et al., 2019; Gan et al., 2019; Masonbrink et al., 2019; Nam et al., 2017). Samtools faidx was used to extract the region of scaffolds containing the tblastn hits and 20,000 base pairs upstream and downstream of the hit. We predicted the exonic sequences of the sp18 and lysin paralogs from these extracted regions using the Protein2Genome command of the program Exonerate version 2.2.0 (Slater \& Birney, 2005). The top scoring prediction from Exonerate was used to define the paralog's exons. We used the same lysin and sp18 sequences from the tblastn search as query sequences. For all full-length sequences, the SignalP-5.0 prediction server was used to predict presence of functional signal peptides (Almagro Armenteros et al., 2019). Presence of signal peptides were predicted with probabilities $>0.9$; the signal peptide cleavage site was predicted with a probability $>0.5$.

\section{Phylogenetic Analysis}

The phylogenetic inference tool RAxML-NG was used to construct all phylogenetic trees with the LG substitution matrix (Kozlov, Darriba, Flouri, Morel, \& Stamatakis, 2019; Le \& Gascuel, 2008). RaxML-NG conducts maximum likelihood based phylogenetic inference and provides branch support using non-parametric bootstrapping (Kozlov et al., 2019). The best scoring topology of 20 starting trees (10 random and 10 parsimony-based) was chosen. RaxML-NG was used to perform non-parametric bootstrapping with 1000 re-samplings that were used to re-infer a tree for each bootstrap replicate MSA. Finally, we mapped the bootstrap scores on the bestscoring starting tree. The Transfer Bootstrap Expectation (TBE) was used as a branch support metric (Lemoine et al., 2018).

DNA multiple sequence alignments (MSA) for phylogenies of lysin and sp18 and their respective paralogs were constructed. First the protein sequences of the genes were aligned using PROMALS3D (Pei, Kim, \& Grishin, 2008; Pei, Tang, \& Grishin, 2008). PROMALS3D uses protein three-dimensional protein structures to inform protein alignments, and representative PDBS for lysin (5UTG) and sp18 (1GAK) were specified (Kresge et al., 2000; D. B. Wilburn et al., 2018). The protein MSAs were used to create DNA alignments of the same genes using the Pal2Nal sever (Suyama, Torrents, \& Bork, 2006). For phylogenetic analysis of $H$. rufescens and $H$. tuberculata VEZP sequences, the protein sequences of the C-terminal ZP modules of each of the proteins were aligned using PROMALS3D (Pei, Tang, et al., 2008).

\section{Syntenic Comparison between Haliotis rufescens and $\boldsymbol{H}$. rubra}

The published genome of Haliotis rufescens is annotated with ORFs identified via transcriptomic sequencing (Masonbrink et al., 2019). We collected the sequences of 2-3 large annotated ORFs surrounding lysin, sp18, and their newly described paralogs within the $H$. rufescens genome. We used these sequences as BLAST queries against the $H$. rubra genome (Gan et al., 2019). The top hits for the $H$. rufescens ORFs were annotated onto the $H$. rubra genome and used to establish synteny between $H$. rubra scaffold 62 and the $H$. rufescens scaffolds 48 and 101 . A 
reciprocal blast of the regions identified as orthologous ORFs in $H$. rubra were queried against the $H$. rufescens genome to verify orthology.

\section{Detecting Selection and Positively Selected Sites}

Values of $d_{N} / d_{s}$ for genes were estimated using the codeml program of PAML 4.8 (Yang, 2007). We compared models of selection using a likelihood ratio test (LRT) between neutral models and models with positive selection. Specifically, we compared M1 v. M2, M7 v. M8, and M8a v. M8. Likelihood ratio tests were performed where the likelihood ratio (LRT) statistic was twice the negative difference in likelihoods between nested models. For M1a v M2a or M7 v Model 8 the LRT was compared to the $\chi^{2}$ distribution with 2 degrees of freedom (Yang, 2007). For the M8a v. M8 comparison, twice the negative difference in likelihoods between the nested models being compared, the LRT statistic, is approximated by the 50-50 mixture distribution of 0 and $\chi^{2}$ with degree of freedom 1 (Swanson et al., 2003). To identify specific sites in proteins evolving under positive selection, we used Naive Empirical Bayes $(\operatorname{Pr}($ omega $>1)=0.95)$. The sites under positive selection were identified using Model 8.

\section{Testing for divergence in regions undergoing positive selection in duplicate sperm proteins}

We designed three unbiased tests to determine if sites under positive selection in either sp18 or sp18-dup are differentially clustered between paralogs. First, we created a parametric test based on the Wald-Wolfowitz runs test (Magel, 1997) to determine whether positively selected sites in the paralogs sp18 and sp18-dup were non-randomly distributed in a protein alignment of both paralogs. The Wald-Wolfowitz runs test determines the randomness of a two-category data string by examining changes between categories by counting "runs." We designed a parametric version of the test to allow the inclusion of three categories. The categories were sites under positive selection in sp18, sites under selection in sp18-dup, and sites under selection in both. The order in which these sites under selection in the categories appeared in an alignment of $H$. fulgens sp18 and $H$. sorenseni sp18-dup became our data string. For the data string generated from our paralog alignment we counted how many times the identity of sites in the string changed plus one. A visualization of the pipeline for preparing this data string and counting "runs" is shown in Supplementary Figure 1A.

To make a parametric version of this runs test, we generated 1000 simulated data strings via bootstrapping based on the proportion of sites shown to be under positive selection in either paralog. For each of these simulated data strings we also calculated the number of runs. The number of data strings with a count of "runs" less than or equal to the count of "runs" found in the data string derived from the paralog protein alignment divided by the total number of simulated data strings gives the parametric probability that by random chance categories of sites would be more or equally clustered compared to the true clustering observed. We also performed a version of the test where sites under selection in both paralogs were eliminated from the analysis. When these sites were eliminated the parametric runs test retained statistical significance ( $p$-value $<0.001)$.

For our second test we evaluated whether sites under positive selection in sp18 vs sp18-dup were distributed throughout the sp18 crystal structure (1GAK) in a significantly different way. In MATLAB Online version 9.9, we identified the plane of best fit between the c-alpha carbons (the first carbon attached to the functional group of an amino acid) of the sp18 crystal structure using linear regression (Supplementary Figure 2A). This plane divided the sp18 crystal structure into 
two sides that we arbitrarily designated "left" and "right" (Supplementary Figure 2B). We mapped the 43 sites in sp18 and the 33 sites in sp18-dup that are under positive selection onto the sp18 crystal structure. Given the number of amino acids sites in each side of the crystal structure, we estimated the expected number of positively selected sites from each paralog that would be expected to be located on either side as the number of amino acid sites on a side divided by the total number of amino acid sites in the molecule and multiplied by the number of positively selected sites in a sp18 paralog. We used a chi-square test to examine whether the real distribution of sites between categories rejected the null expectation. This test determined whether the distribution of sites under selection in either paralog was not distributed similarly between the sides of the protein. We also created a plane perpendicular to the plane of best fit to divide the sp18 crystal structure into the categories "top" and "bottom" (Supplementary Figure $2 \mathrm{C})$. We repeated an analysis for this new pair of categories that is identical to what was described previously. This analysis gave us a sense if sites under selection in either paralog were clustered nonrandomly throughout the crystal structure in different ways.

For our last clustering test we determined whether sites under positive selection in a paralog were more likely to be close in proximity in three-dimensional space to another site under positive selection in the same paralog rather than a site under positive selection in the other paralog. For each site that was under selection in a paralog, we identified the closest positively selected site in three-dimensional space that belonged to either paralog. We then calculated the expected number of times by chance the closest adjacent site for each site under positive selection would belong to the same gene rather than the other paralog. We used a chi-square test to determine whether observed sites under selection in one paralog were statistically more likely to be close to positively selected sites belonging to the same paralog than what would be expected by chance. This test has four categories of sites (for each paralog the nearest site could belong to the same paralog or not), and therefore three degrees of freedom were used to determine the $p$-value. Sites that were under selection in both paralogs were counted twice in this analysis since these sites were undergoing positive selection independently in both paralogs. When the closest adjacent site to a positively selected site in one paralog was undergoing positive selection in both paralogs, the adjacent site was treated as belonging to the same paralog.

\section{Identification of Sp18 peptides}

H. tuberculata testis tissue was homogenized in $1 \%$ sodium dodecyl sulfate with BME at $70{ }^{\circ} \mathrm{C}$ for 30 minutes. Testis samples were separated by SDS-PAGE using a Tris-Tricine buffering system with discontinuous $4 \%$ resolving $/ 15 \%$ separating acrylamide gels. Samples were electrophoresed at $50 \mathrm{~V}$ for $15 \mathrm{~min}$ followed by $100 \mathrm{~V}$ for $90 \mathrm{~min}$. The gel was run with the BioRad Broad Range Ladder and stained with Coomassie Blue R-250 for 15 minutes. Using the ladder as reference, the lysin and sp18-containing region ( 14-22 kDa) of the polyacrylamide gel was excised using a clean scalpel, with multiple rounds of perfusion with an ammonium bicarbonate solution followed by acetonitrile to extract detergents and salts. Trypsin proteolysis of immobilized proteins was by perfusion of a Trypsin solution (40 ug/ml stock Trypsin 1:10 in 50 $\mathrm{mM}$ ammonium bicarbonate) and incubation at $37^{\circ} \mathrm{C}$ overnight. The supernatant from the digest was collected along with the supernatant from two rounds of hydration with ammonium bicarbonate and extraction with $50 \%$ acetonitrile. The collected supernatant containing the liberated peptides was concentrated to a dry pellet using a vacuum centrifuge then reconstituted in $0.1 \% \mathrm{FA}$ for liquid chromatography tandem mass spectrometry (LC/MS-MS). Unique peptides for sp18 copy \#1 were identified in the sample using the Crux toolkit comet command (Park, Klammer, Kall, MacCoss, \& Noble, 2008). The protein sequence database was composed of a six-frame translation of the $H$. tuberculata testis transcriptome. 


\section{Identification of ZP Proteins}

An exhaustive BLAST search of the $H$. tuberculata ovary transcriptome identified all cDNA sequences with homology to $H$. rufescens VEZPs. A previous study used a similar approach to originally identify known VEZPs in $H$. rufescens indicating that this approach should be sufficient to identify novel VEZPs (Aagaard et al., 2010). All cDNA sequences that matched VEZPs were filtered for duplicates using CD-HIT-EST with a threshold of 0.9 sequence identity (Huang, Niu, $\mathrm{Gao}, \mathrm{Fu}, \& \mathrm{Li}, 2010)$. The longest sequence from each cluster created by CD-HIT-EST was chosen as the cluster's representative sequence. All $H$. tuberculata sequences from this filtering process were translated and the C-terminal ZP modules were identified by identifying conserved cysteine residues. The ZP module protein sequences from both $H$. tuberculata and $H$. rufescens were aligned using PROMALS3D (Pei, Tang, et al., 2008). The MSA of these ZP modules from were used to construct a VEZP homolog protein phylogeny using the same RAXML-NG protocol described above for lysin and sp18 paralog phylogenies. New sequences were uploaded to Genbank under accession numbers OK491878- OK491909.

\section{Results}

\section{Genomic Analysis Reveals Tandem Duplications of Ancestral Abalone Acrosomal Proteins}

By pairing phylogenetic and genomic analysis of abalone species belonging to the North American clade $(H$. rufescens, $H$. sorenseni, $H$. discus) and the Australian clade $(H$. rubra, $H$. laevigata), we identifed ancestral duplications of both sp18 and lysin (Figure 1; sp18-dup and lysin-dup, respectively). We calculated maximum likelihood DNA phylogenies independently for lysin and sp18 with their paralogs and rooted the phylogenies by orthology (Figure 1A \& 1B). Predicted intron/exon boundaries of the novel acrosomal protein paralogs were shared with lysin and sp18 (Metz, Robles-Sikisaka, \& Vacquier, 1998). No mutations causing pseudogenization were detected within the predicted CDS of either paralog. For the abalone species with published genomes, only one (H. rufescens) has a published testes transcriptome (Palmer et al., 2013). Full-length sequences of lysin, sp18, and sp18-dup are expressed in the testes transcriptome of $H$. rufescens; however, lysin-dup was not detected.

Sequence analysis is consistent with the sp18-dup gene encoding a functional reproductive protein ancestral to Haliotis. Sp18-dup is predicted to have a signal peptide sequence and maintains a pair of cysteine residues involved in forming a structurally important disulfide-bond in sp18 (Kresge et al., 2000, 2001). Sp18-dup has not been identified in previous analysis due to the high divergence between it and sp18 (27.5\% sequence identity between $H$. rufescens sp18 paralogs) obscuring homology.

Lysin-dup was identified in all abalone genomes investigated but was not detected in the testes illumina transcriptome of $H$. rufescens (Palmer et al., 2013). The absence of lysin-dup in the testes transcriptome could indicate insufficient read depth, differences in tissue-expression, or potentially pseudogenization. Since the full-length sequence of lysin-dup was not identified within the $H$. rufescens testis transcriptome, lysin sequences were used instead to identify lysindup exons within abalone genomes. However, divergence between lysin and lysin-dup likely prevented the identification of full-length coding sequence from abalone genomes. Only exons 2-4 could be identified ( $79 \%$ of query sequence) within $H$. rufescens and $H$.rubra. The missing exons 1 and 5 contain the signal peptide and the $\mathrm{N}$ - and $\mathrm{C}$-termini of the molecule. In lysin, the $\mathrm{N}$ - and $\mathrm{C}$-terminus are under strong positive selection promoting extensive divergence that 
reduces the ability to identify these exons using homology-based approaches (Lee et al., 1995; Lyon \& Vacquier, 1999).

In the Australian abalone genomes lysin, lysin-dup, sp18, and sp18-dup are all found within a single contig with $233 \mathrm{~kb}$ separating the paralog pair of lysin and lysin-dup from the paralog pair of sp18 and sp18-dup. But in the genome of the North American abalone species $H$. rufescens, the paralog pair of lysin and lysin-dup are on a separate scaffold from the paralog pair of sp18 and sp18-dup. We compared the Australian contig containing the four acrosomal protein paralogs with the two $H$. rufescens contigs containing the lysin and sp18 paralog pairs respectively (Figure 1C). We found several ORFs surrounding each paralog pair in $H$. rufescens that were found in the same order between in $H$. rubra, indicating synteny between scaffolds. All four acrosomal proteins being located near each other in the same scaffold in the $H$. rubra genome suggests that tandem duplication led to recurrent duplications of this protein family (Reams \& Roth, 2015). The sp18 ORF codes in a different direction than the other paralogs, suggesting that transposition and inversion may have also contributed to duplications within this protein family (Reams \& Roth, 2015).

\section{Patterns of divergence of ancestral acrosomal protein paralogs indicate subfunctionalization}

Lysin, sp18, and sp18-dup all contained sites detected to be subjected to positive selection. (Table 1). Lysin-dup did not show signatures of positive selection, though this could be due to having insufficient sequences to provide the statistical power to conduct the test (Table 1) (Anisimova, Bielawski, \& Yang, 2001). Clustering and distribution of amino acid sites undergoing positive selection can identify regions important to the function of rapidly evolving genes (Anisimova et al., 2001). For example, many of the sites in lysin that are undergoing positive selection (11/23) are in a region of the molecule that binds its egg receptor VERL (D. B. Wilburn et al., 2018). We investigated the distribution of sites undergoing positive selection in sp18 and sp18-dup. Similar regions of the molecule undergoing positive selection in both paralogs would suggest a shared biochemical mechanism while differences in distributions of positively selected sites would indicate divergence in biochemical mechanism.

By mapping sites under positive selection onto a protein alignment of sp18 and sp18-dup, we determined that sites under positive selection in either paralog are non-randomly distributed across the protein alignment and differentially clustered. We analyzed the selected sites in the primary sequence alignment with a parametric adaptation of the runs test (Wald-Wolfowitz test) (Figure 2A) (Magel, 1997). This analysis showed that there were significant runs of sites undergoing positive selection in either paralog ( $p$-value $=0.026)$, consistent with different regions evolving under positive selection among paralogs. Positive selection acting on different regions of the protein alignment is consistent with subfunctionalization of paralogs.

We investigated clustering of positively selected sites in three-dimensional space. By mapping sp18 and sp18-dup positively selected sites onto the sp18 structure, it is visually apparent that there are distinct clusters of sites under selection between paralogs (Figure 2B). Using the plane of best fit through the crystal structure we divided the molecule into "left" and "right" sides agnostic to the location of positively selected sites. To define the "top" and "bottom" of the molecule we used a plane perpendicular to the plane of best fit. Sites under positive selection in sp18-dup were statistically more likely to be on the "right" side than on the "left" ( $p$-value <0.05) (Supplementary Figure 2). Sp18 sites, using the same test, showed no statistically significant difference from the null distribution. However, sites under positive selection in sp18 were enriched on the "top" of the molecule rather than the "bottom" ( $p$-value $<0.05)$ while the 
distribution of sp18-dup positively selected sites did not significantly differ (Supplementary Figure 2). These two tests show that sites under selection in sp18 and sp18-dup are distributed differently across their three-dimensional structures.

We also developed a test to examine whether sites under selection in sp18 and sp18-dup were statistically more likely to be adjacent to a site under selection from the same paralog. Such a pattern of clustering would indicate a spatial relationship between positively selected sites belonging to a particular paralog. For each site under positive selection in sp18 or sp18-dup, we identified whether the closest positively selected site in three-dimensional space was significantly more likely to belong to the same paralog. We found that sites under selection in both paralogs were more likely to have the closest positively selected site belong to the same paralog rather than the other paralog according to a chi-squared test ( $p$-value $<0.01$ ). Together, the runs test analysis and three-dimensional analyses point to diversifying selection postduplication of these proteins to promote functional diversification.

Because Lysin-dup was not detected to be under positive selection, we did not test for differences in sites under selection between lysin paralogs. However, we did evaluate how the lysin-dup sequence diverged from lysin. Many of the sites shown to be undergoing positive selection in lysin differ in sequence from lysin-dup (13/14) when comparing the $H$. rufescens sequences (Figure 3B). Although this comparison is not significant $(p=0.088)$, this suggests similar sites driving the diversification in sequence of lysin between species and between lysin and its paralog lysin-dup.

\section{H. tuberculata sp18 and lysin duplications are species-specific}

Previous work described a lysin duplication unique to $H$. tuberculata (Clark et al., 2007). The lysin paralogs were shown to be evolving under positive selection and to be maintained in the testis proteome (Clark et al., 2007). Sites that vary between European lysin paralogs are largely located on the face of the molecules interacting with lysin receptor VERL (Figure 3A). To investigate the presence of additional sp18 and lysin paralogs in H. tuberculata, we constructed a long-read PacBio testis transcriptome. Performing tBLASTN searches of the $H$. tuberculata transcriptome for lysin and sp18 revealed the previously described species-specific duplication of lysin $(H$. tuberculata lysin copy \#1 and copy \#2) and a novel duplication of sp18 $(H$. tuberculata sp18 copy \#1 and copy \#2).

Phylogenetic analysis indicates the $H$. tuberculata sp18 paralogs are the result of a recent duplication and not ancestral to Haliotis. Sequence information from other abalone species is needed to determine whether this duplication is species-specific to $H$. tuberculata; it appears to be specific to the abalone clade containing the European species. The signal sequences of the $H$. tuberculata sp18 paralogs are more similar to each other than to signal sequences from other species' sp18 paralogs. Signal sequences are not part of the mature protein and not subjected to the same evolutionary pressures driving rapid divergence, therefore these sequences show more conservation between closely related paralogs. This similarity in signal sequence between $H$. tuberculata sp18 paralogs (15/19 sites are identical) further supports that these paralogs are the result of a non-ancestral duplication. Despite being a more recent duplication of sp18, the paralogs have a low sequence identity (39\%), lower than that of the $H$. tuberculata lysin paralogs $(83 \%)$. This is consistent with sp18 having a higher $d_{N} / d_{S}$ and evolving more rapidly than lysin (Table 1). H. tuberculata sp18 paralogs maintain a pair of structurally important cysteine residues involved in forming a disulfide bond. Rapid sequence divergence, no premature stop codons, and both genes being expressed in the testis transcriptome are all 
indicators that both sp18 paralogs (referred to here as H. tuberculata copy \#1 and copy \#2) are likely to be functional.

The $H$. tuberculata sp18 copy \#1 is the more divergent to the ancestral sequence than copy \#2, as indicated by its long branch in the sp18 phylogeny (Figure 1B). When comparing the sequence identity of $H$. tuberculata sp18 paralogs to $H$. rubra sp18 (an outgroup sequence), copy \#1 shows a lower sequence identity (41\%) than copy \#2 (69\%). This rapid sequence divergence of copy \#1 without accruing mutations causing pseudogenization suggests strong positive selection. However pairwise $d_{N} d_{s}$ between $H$. tuberculata sp18 paralogs could not be reliably estimated due to extensive divergence resulting in saturation (multiple substitutions per site) (Swanson \& Vacquier, 1995). Maintenance of both paralogs in the testis proteome despite the observed sequence divergence would indicate that both paralogs are being selected for functions, presumably related to fertilization. We used data dependent acquisition mass spectrometry to identify peptides belonging to either paralog in the $H$. tuberculata testes proteome. Diagnostic peptides were detected for copy \#1 but not copy \#2. Despite being the more divergent sp18 sequence, copy \#1 is maintained in the proteome. This result indicates that copy \#1 is likely important for fulfilling sp18's membrane fusion function in $H$. tuberculata. I

\section{Lack of Recent Duplications of Egg Coat Proteins}

We generated an ovary PacBio transcriptome for $H$. tuberculata to identify VEZP proteins. Using the 33 VEZP and ZP-domain sequences from the $H$. rufescens ovary transcriptome as the initial query sequences, exhaustive tBlastn searches of the ovary transcriptome were used to identify all cDNA sequences with sequence similarity to any $H$. rufescens VEZP. ZP module protein sequences were extracted from our $H$. tuberculata cDNA hits and the $33 H$. rufescens VEZPs and then were aligned to construct a phylogeny (Figure 4). Clustering of $H$. tuberculata and $H$. rufescens $\mathrm{ZP}$ module sequences indicate that these distantly related abalone species have the same complement of ZP-proteins in their transcriptomes. In H. tuberculata's ovary transcriptome, orthologs of 32 of the $33 \mathrm{H}$. rufescens ovary ZP-domain proteins were identified. No VEZPs, including VERL and its most closely related paralogs VEZP14 and VEZP9 were duplicated. The only missing sequence belonged to ZPC, a gene whose cDNA sequence contains a premature stop codon in $H$. rufescens and for which no peptides were detected in the $H$. rufescens VE proteome (Aagaard et al., 2010). Therefore, ZPC is likely pseudogenized in $H$. rufescens and its expression its expression is no longer maintained in European abalone.

Remarkably, no new ZP-module-containing proteins were identified in $H$. tuberculata despite the species having multiple clade-specific duplications of acrosomal proteins. These results suggest that the clade-specific maintenance of duplicated sperm acrosomal proteins found in the European abalone $H$. tuberculata are unlikely to be the result of duplicated egg proteins.

\section{Discussion}

Despite decades of research examining the evolution of abalone fertilization genes, only recently have genomic resources been available that enable a broad investigation into the evolution of the protein families to which lysin and VERL belong. Here, we explore the contributions of duplication and sequence divergence to the evolution of abalone fertilization genes across the genus Haliotis. For our investigation we generated ovary and testes transcriptomes from the European abalone H. tuberculata and utilized recently published North American and Australian abalone genomes and a North American abalone testes transcriptome (Palmer, McDowall et al. 2013, Nam, Kwak et al. 2017, Botwright, Zhao et al. 2019, Gan, Tan et al. 2019, Masonbrink, Purcell et al. 2019). We discovered novel duplications of both lysin and 
sp18 ancestral to abalone, indicating that abalone lysin and sp18 are members of an ancestral abalone protein family with four members. The newly discovered sp18 paralog (sp18-dup) was shown to be undergoing positive selection, like lysin and sp18, and expressed in the testes of North American abalone. Further, differences in clustering of positively selected sites in sp18 compared to sp18-dup is consistent with models of subfunctionalization. We investigated whether there are clade-specific duplications of abalone VEZPs or acrosomal proteins. In addition to a species-specific lysin duplication described in a previous publication (Clark et al., 2007), the $H$. tuberculata testes transcriptome contains a clade-specific duplication of sp18 not found in Australian or North American abalone species. However, no duplications of VERL or other VEZPs were observed between North American or European abalone, indicating that VEZP gene content is conserved across the genus Haliotis. Together, this data demonstrates that recurrent duplication and diversification driven by positive selection drives the evolution of an acrosomal protein family involved in fertilization in Haliotis.

\section{Recurrent Duplication and Positive Selection of Acrosomal Proteins in Abalone}

In the $H$. rubra abalone genome, the paralogs lysin, lysin-dup, sp18, and sp18-dup are found on a single scaffold. This clustering within the genome indicates that ancestral tandem duplication events occurred leading to the creation of this acrosomal protein family (Reams \& Roth, 2015). Further, three of the four ancestral paralogs were shown to be maintained in the testis transcriptome and to be evolving under positive selection, a common characteristic of reproductive proteins.

This evolutionary pattern of duplication paired with sequence diversification found in the abalone acrosomal protein family can be compared to protein families in other taxa which contain sperm proteins mediating fertilization. Notably, the mammalian Izumo gene family contains four ancestral paralogs whose members all show testes-specific tissue expression in humans (Grayson \& Civetta, 2012). Izumo1 is an essential gene for sperm-egg plasma membrane fusion in mammals that functions by binding the egg plasma membrane protein JUNO (Bianchi et al., 2014). There is evidence that the other three Izumo paralogs may also possess important, although potentially varied, functions in fertility (Ellerman et al., 2009). All four paralogs have been shown to be undergoing rapid sequence evolution in at least one mammalian lineage, for Izumo1, 2, and 3 this is driven by positive selection and for Izumo4 this appears to be driven by relaxed selection (Grayson, 2015; Grayson \& Civetta, 2012). Given that both the abalone acrosomal protein family and the mammalian Izumo family both contain multiple paralogs showing testis-specific function, subfunctionalization may be a common driver of the evolution of fertilization and reproductive genes across taxa. Understanding how fertilization proteins emerge and evolve can be important for identifying and understanding mechanisms of fertilization across diverse taxa.

\section{Recurrent Subfunctionalization of Abalone Acrosomal Proteins}

Differences in optimal mating rates for sperm and eggs can drive antagonistic coevolution of reproductive proteins. Under this sexual conflict scenario, evolution of egg coat proteins interacting with sperm acrosomal proteins could lead to constrained evolution on the sperm side (Gavrilets \& Waxman, 2002). Duplication followed by diversification of sperm fertilization proteins can be an important means of sperm escaping evolutionary constraints imposed by egg protein evolution. For two duplication events within the abalone acrosomal protein family there is evidence of subfunctionalization from either functional experiments (lysin vs. sp18, (Kresge et al., 2001; Swanson \& Vacquier, 1997) or site-clustering analysis (sp18 vs. sp18-dup, current manuscript). 
Plasma membrane fusion in fertilization or other contexts is traditionally thought to consist of two steps, binding and fusion (Bianchi \& Wright, 2020). In sea urchins, both steps are mediated by different regions of the same protein, su-bindin (Ulrich, Otter, Glabe, \& Hoekstra, 1998; Vacquier \& Moy, 1977; Vacquier \& Swanson, 2011). However, in abalone these steps may have been partitioned between sp18 and sp18-dup via subfunctionalization. Abalone eggs have a thin layer directly overlaying the surface of the plasma membrane which morphologically resembles a duplication of the elevated VE (Mozingo et al., 1995). Just as lysin binds the VE protein VERL, sp18 may bind a VEZP protein found within the thin layer overlaying the abalone egg plasma membrane. Indeed, in addition, to having a strong fusagenic function, sp18 has been demonstrated to bind to VEZP proteins, an unsurprising trait for a lysin paralog (Aagaard et al., 2010). One possibility is that the subfunctionalization of sp18 and sp18-dup may have been driven by the separation of the steps of plasma membrane binding and fusion between paralogs.

While the paralogs lysin-dup and lysin do show high sequence divergence, only lysin shows evidence of positive selection. Therefore, the observed sequence divergence is likely driven by lysin's evolution post-duplication. Unlike the other acrosomal protein family paralogs discussed in this paper, lysin-dup is not detected in the testis transcriptome. However, there is an appealing hypothesis as to its potential function. In abalone egg coats there are two VEZP proteins capable of binding lysin, VERL the major binding partner of lysin and VEZP-14 the most recent paralog of VERL (Aagaard et al., 2013). It is possible that lysin-dup may be the binding partner of VEZP-14 and if true this could explain why lysin shows correlated evolution with VERL but not VEZP-14 (Aagaard et al., 2013). Currently there is insufficient data to test for correlated rates of evolution between lysin-dup and VEZP-14. However, further molecular and biochemical characterization through binding kinetic analysis could test the hypothesis that lysin-dup and VEZP-14 interact.

\section{Species-Specific Duplications of Acrosomal Proteins in Abalone}

Previous work described a lysin duplication unique to $H$. tuberculata and maintained in the testis proteome (Clark et al., 2007). In this study a clade-specific duplication of sp18 was discovered within the $H$. tuberculata transcriptome. Despite having two acrosomal protein duplications, European abalone's ovary transcriptome did not reveal any novel VEZP protein sequences indicative of a duplication event. While gene duplications are an ongoing contributor to the evolution of sperm fertilization genes in abalone, this may not be true for egg fertilization genes. Our data suggests that it is not duplications on the egg side driving the duplication of abalone acrosomal proteins in $H$. tuberculata. This could be explained by different selective pressures on the sperm and the egg, such as sperm competition and polyspermy risk (Carlisle \& Swanson, 2020). Further, It does not seem that a process of gene birth and loss explains the evolution of abalone's acrosomal protein family since all paralogs are maintained in the transcriptome and have accrued no pseudogenizing mutations. A hypothesis for the duplication and diversification of acrosomal protein paralogs in $\mathrm{H}$. tuberculata is that paralogs are specialized for different binding sites of their egg receptor or different allelic variants of their receptor. For example, $H$. rufescens VERL has 22 tandem ZP-N domains with three unique amino acid sequences, $H$. tuberculata VERL may show similar differences in ZP-N sequences and $H$. tuberculata lysin paralogs may be optimized for binding different ZP-N sequences (Galindo, Moy, Swanson, \& Vacquier, 2002). In addition, the abalone $H$. tuberculata VERL may be polymorphic, as seen for $H$. corrugata VERL, and lysin paralogs are optimized for VERL allelic variants (Clark et al., 2009). This study observed that sites that vary between $H$. tuberculata lysin paralogs are largely located on the face of the molecules interacting with lysin receptor VERL (Figure 3A). Unlike 
the distribution of positively selected sites between sp18 and sp18-dup where sites are differentially clustered on the protein structure. This pattern of diversification may be suggestive of specialization of function, such as interacting with different VERL allelic variants or VERL ZP$\mathrm{N}$ domains. Further characterization of VERL in $\mathrm{H}$. tuberculata and population-level variation is necessary to explore these hypotheses.

\section{Conclusion}

This study characterizes duplication events of a sperm acrosomal protein family with functions directly associated with fertilization. Although lysin was one of the first fertilization proteins discovered and the first for which an egg binding partner was defined, its evolutionary origins are unknown. By placing duplication events of lysin and sp18 within their genomic context and identifying clade-specific duplication events, this study has revealed the importance of duplication for the evolution of this protein family that has previously been unknown. We describe six acrosomal protein paralogs arising from both ancestral and clade-specific duplication events (Figure 5). Recurrent duplication events of sperm acrosomal proteins have occurred throughout the evolutionary history of abalone. For the two abalone species with transcriptomic data both have paralogs maintained in the testis transcriptome. Remarkably none of these genes have been pseudogenized and many are undergoing strong positive selection consistent with maintenance of their function in abalone reproduction. Further inquiry is required to investigate why these proteins are undergoing duplication, the functional consequences of these duplication events, and whether other fertilization proteins in other species (as also seen for the mammalian Izumo family) are undergoing recurrent duplication events.

\section{Author Contributions}

JAC and WJS designed the research. JAC and MG performed the research. JAC and WJS analyzed the data. JAC wrote the paper.

\section{Acknowledgements}

This study was supported by NIH Grant HD076862 to Willie J. Swanson and a National Science Foundation Graduate Research Fellowship to Jolie A. Carlisle. Megan Glenski was supported by University of Washington School of Medicine-Gonzaga University Regional Health Partnership. Thank you to France Haliotis for help acquiring $H$. tuberculata samples, to Evan Cox, Dr. Daniel Promislow, Dr. Josh Schraiber, and Dr. Damien Wilburn for help with data analysis, and to Alberto Rivera, Dr. Jan Aagaard, and Dr. Bryce Taylor for useful discussions and comments. All research was performed on the traditional lands of the Duwamish Tribe. To learn more about the Duwamish Tribe and their continuing legacy, please visit https://www.duwamishtribe.org/.

\section{Conflicts of Interest}

The authors report no conflicts of interest 


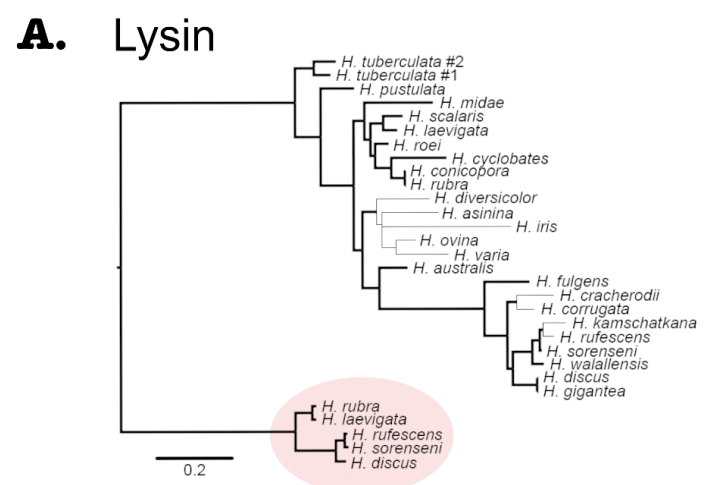

\section{B. SP18}

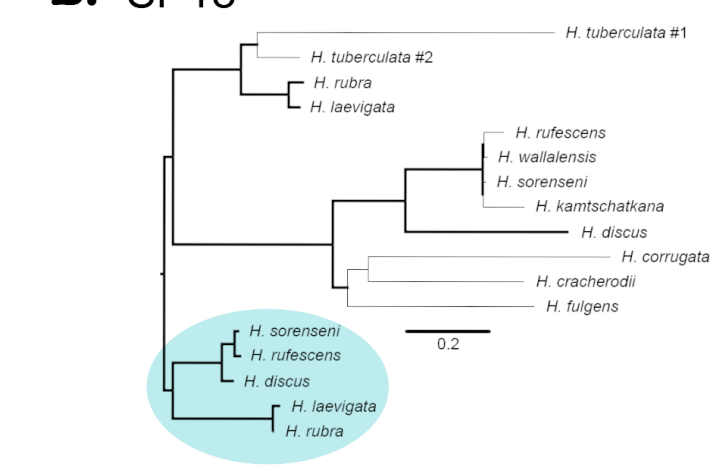

C. Haliotis rufescens

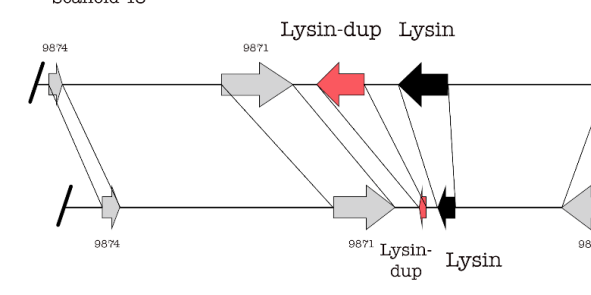

Scaffold 62 Haliotis rubra

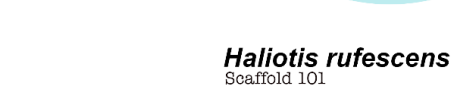

$\underset{\text { Scalfold } 101}{\text { Haliot rufescens }}$

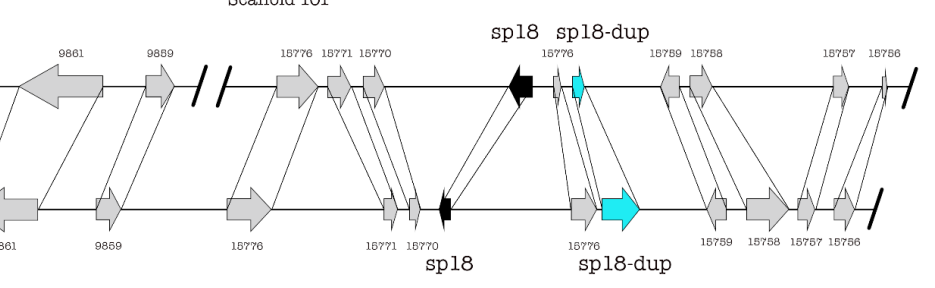

$-50 \mathrm{~kb}$

Figure 1: Syntenic and phylogenetic analysis indicate that four tandem acrosomal proteins are ancestral to all abalone.

A. Ancestral duplication led to the paralogs lysin and lysin-dup (Red). Bold lines indicate greater than $80 \%$ bootstrap branch support. B. Ancestral duplication led to the paralogs sp18 and sp18-dup (blue). A clade specific duplication of sp18 is present in the $H$. tuberculata testes transcriptome. Bold lines indicate greater than $60 \%$ bootstrap branch support. C. Lysin (black), sp18 (black) and their paralogs lysin-dup (red) and sp18-dup (blue) are found in the genomes of North American and Australian abalone. Syntenic analysis indicates that paralogs are located near each other in the abalone genome, although all four paralogs are only genetically linked in Australian abalone genome assemblies. In the North American abalone H. rufescens genome assembly, lysin and sp18 are found on separate scaffolds linked with their paralogs. 

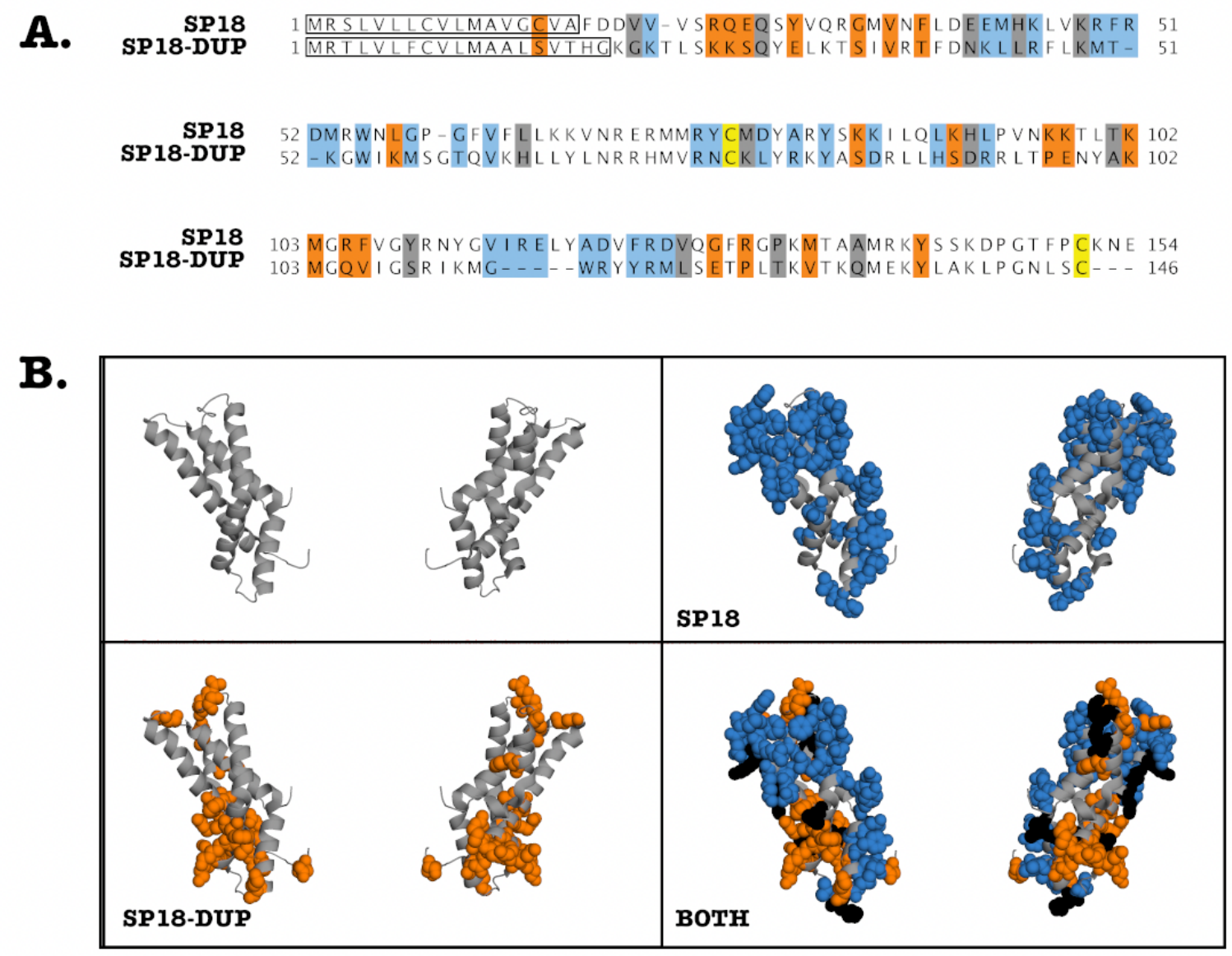

Figure 2: Clustering of positively selected sites is consistent with subfunctionalization of SP18 and SP18-dup.

A. Alignment of $H$. fulgens sp18 and $H$. sorenseni sp18-dup mature protein sequences. Sites under positive selection are highlighted. Blue (Sp18), Orange (Sp18-dup), and Black (both). A modified parametric runs test was used to determine that there were statistically significant runs in linear sequence space of sites under positive selection in sp18 or sp18-dup (p-value - 0.0XX). B. Sites under positive selection in sp18 or sp18-dup were mapped onto the crystal structure of sp18 from $H$. fulgens and appear clustered in 3-D space. 
A.
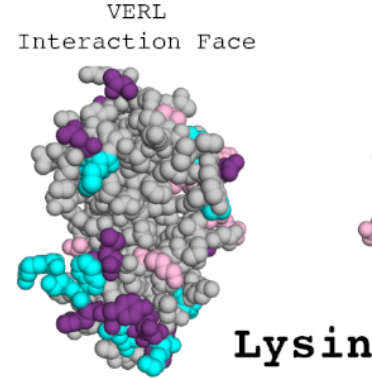

C.

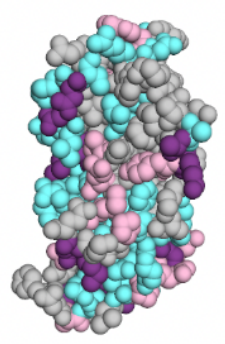

SP18

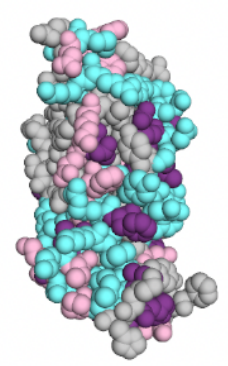

B.

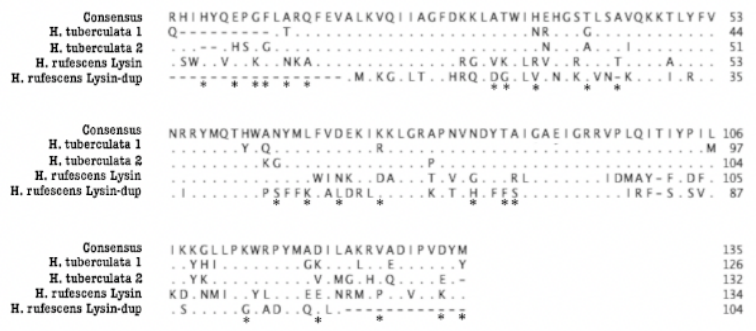

D.

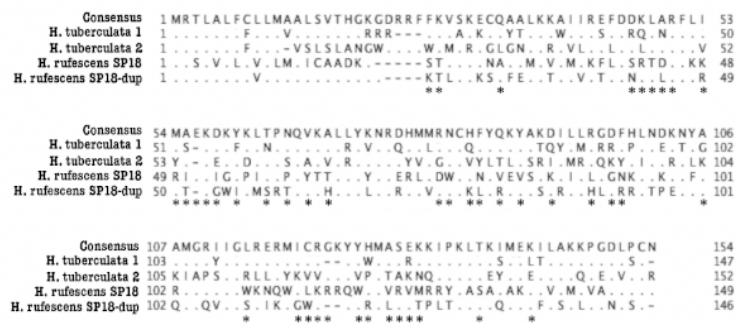

Figure 3. Duplication and divergence of lysin and sp18 paralogs in $H$. tuberculata.

A.) Sites that differentiate $H$. tuberculata lysin paralogs from $H$. rufescens lysin are mapped onto the $H$. rufescens NMR structure (PDB 5UTG). Blue - Sites that differ between $H$. rufescens lysin and $H$. tuberculata Copy \#1, Pink - Sites that differ between $H$. rufescens lysin and $H$. tuberculata Copy \#2, Purple - Sites that differ between $H$. rufescens lysin and both $H$. tuberculata lysin paralogs. Notably the face of the lysin molecule that interacts with VERL harbors most substitutions between $H$. tuberculata paralogs, suggestive of a shared interaction face for both $H$. tuberculata lysin paralogs. B.) Alignment of Lysin paralogs in $H$. rufescens and H. tuberculata. Both sets of paralogs arose from independent duplication events. Asterisks indicate sites that are shown to be under positive selection in $H$. rufescens lysin. C.) Sites that differ between $H$. tuberculata sp18 paralogs are mapped onto the crystal structure of sp18 from $H$. fulgens (PDB 1GAK). Blue - Sites that differ between $H$. rufescens sp18 and $H$. tuberculata Copy \#1, Pink - Sites that differ between H. rufescens sp18 and H. tuberculata Copy \#2, Purple - Sites that differ between $H$. rufescens sp18 and both $\mathrm{H}$. tuberculata sp18 paralogs. D.) Alignment of sp18 paralogs in $H$. rufescens and $H$. tuberculata. Both sets of paralogs arose from independent duplication events. Asterisks indicate sites that are shown to be under positive selection in $H$. rufescens sp18. 


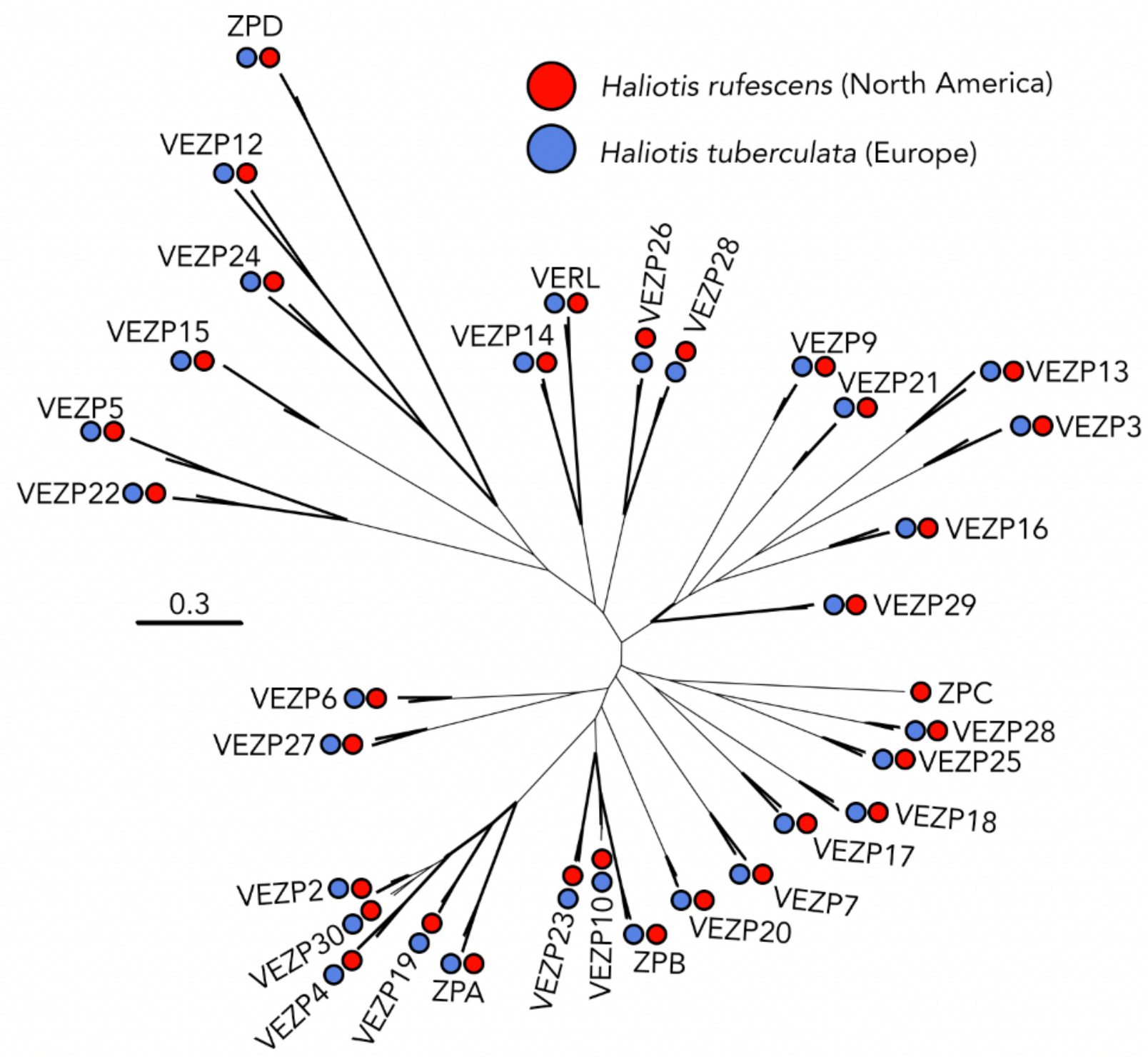

Figure 4. VEZP proteins are conserved across abalone species.

The distantly related abalone species $H$. rufescens (Red) and $H$. tuberculata (Blue) share the same vitelline envelope zona pellucida (VEZP) proteins within their ovary transcriptomes. Although there are species-specific duplications of lysin and sp18 in $\mathrm{H}$. tuberculata, there is no evidence of species-specific duplications of VERL or other VEZP proteins. Bold lines indicate greater than $80 \%$ bootstrap branch support. 


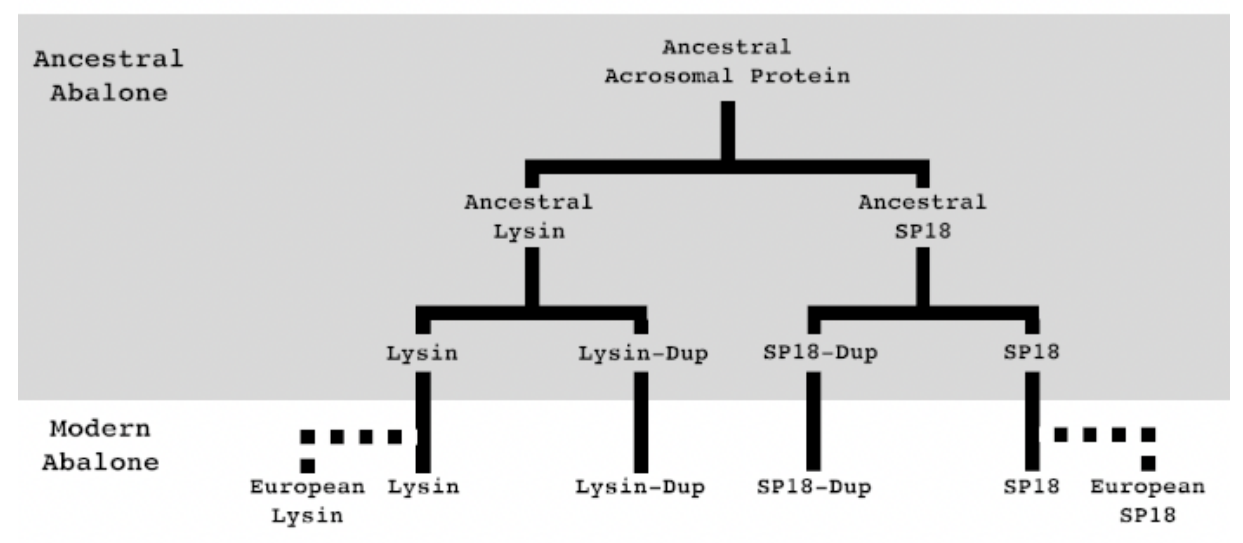

Figure 5: Summary of abalone acrosomal duplications.

Abalone acrosomal proteins have duplicated multiple times over the course of abalone evolution. Five duplication events can describe the currently identified protein family. Most duplications are ancestral to all abalone, but species-specific duplications in European abalone point to duplication paired with positive selection still playing an important role in the evolution of abalone fertilization pathways. Solid lines indicate orthologs present in all abalone, dotted lines indicate species-specific paralogs. 


\begin{tabular}{|l|l|l|l|l|}
\hline Gene & Model & $-2 \Delta I$ & $d_{N} / d_{s}$ & $\begin{array}{l}\text { \% Positively } \\
\text { Selected Sites }\end{array}$ \\
\hline Sp18 & M1a vs M2a & $84.2^{* *}$ & 4.4 & 48 \\
\hline & M7 vs M8 & $95.3^{* *}$ & 4.2 & 48 \\
\hline $\begin{array}{l}\text { Sp18-sup (do } \\
\text { you man } \\
\text { “dup”?) }\end{array}$ & M8a v M8 & $84.3^{* *}$ & 4.2 & 48 \\
\hline & M1a vs M2a & 4.1 & & - \\
\hline Lysin-dup & M7 vs M8 & 4.1 & - & - \\
\hline & M8a v M8 & $4.1^{*}$ & - & 49 \\
\hline & M7 vs M8 & 1.5 & 1.8 & - \\
\hline Lysin & M8a v M8 & 1.3 & - & - \\
\hline & M1a vs M2a & $156.1^{* *}$ & 1.2 & - \\
\hline & M7 vs M8 & $156.7^{* *}$ & 1.1 & 21 \\
\hline & M8a v M8 & $141.1^{* *}$ & 1.1 & 22 \\
\hline
\end{tabular}

Table 1: Acrosomal protein paralogs are evolving under positive selection.

Codon substitution models were used to analyze sequences of sp18, sp18-dup, lysin-dup, and lysin. Site models allowing for several neutral models (M1a, M7, and M8a) or selection models (M2a, M8, and M8a) allowing for variation among sites, were fit to the data using PAML. Sites undergoing positive selection were detected in sp18 and lysin for all model comparisons. A more powerful test (M8a v M8) detected positive selection in sp18-dup as well as sp18 and lysin. Estimates of the likelihood ratio statistic $(-2 \Delta I), d_{N} / d_{s}$, and the percentage of sites that are under positive selection are given. Significant tests are highlighted in yellow. ( ${ }^{*}$, significant at $P<0.05$; ${ }^{* *}$, significant at $P<0.005$.) 


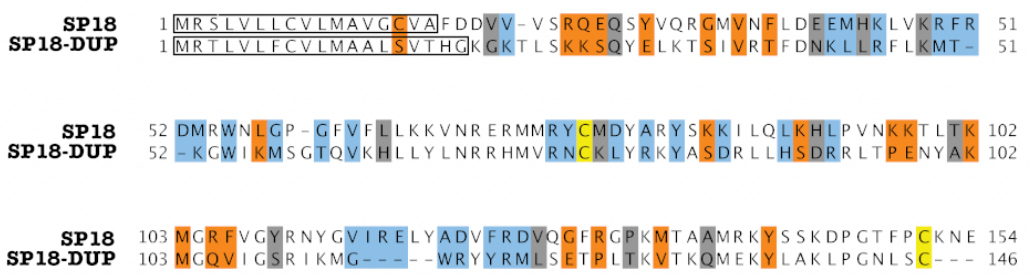

$\begin{aligned} \text { SP18 } & 103 \text { MGRFVGYRNYGVIRELYADVFRDVQGFRGPKMTAAMRKYSSKDPGTFPCKNE } 154 \\ \text { SP18-DUP } & 103 \text { MGQVIGSR I KMG - - -WRYYRMLSETPLTKVTKQMEKYLAKLPGNLSC_.- } 146\end{aligned}$

Create a datastring from the alignment of sites that are under positive selection in either sp18 (blue), sp18-dup (orange), or both (gray).

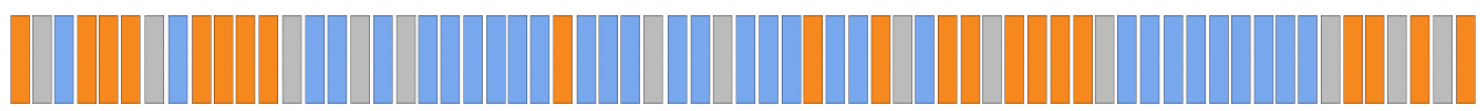

Count the runs of sites from any category in the data string.

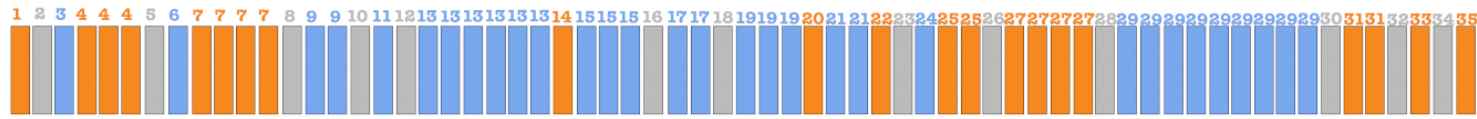

Run Count: 35

\section{Supplementary Figure 1: Calculation of Runs of Positively Selected Sites.}

The Wald-Wolfowitz runs test determines the randomness of a two-category data string by examining changes between categories by counting "runs." We designed a parametric version of the test to allow the inclusion of three categories. The categories were sites under positive selection in sp18, sites under selection in sp18-dup, and sites under selection in both. The order in which these sites under selection in the categories appeared in an alignment of $H$. fulgens sp18 and $H$. sorenseni sp18-dup became our data string. For the data string generated from our paralog alignment we counted how many times the identity of sites in the string changed plus one. A visualization of the pipeline for preparing this data string and counting "runs" is shown here. We also performed an alternate version of this analysis where the gray sites (both) were removed when calculation changes in categories. 

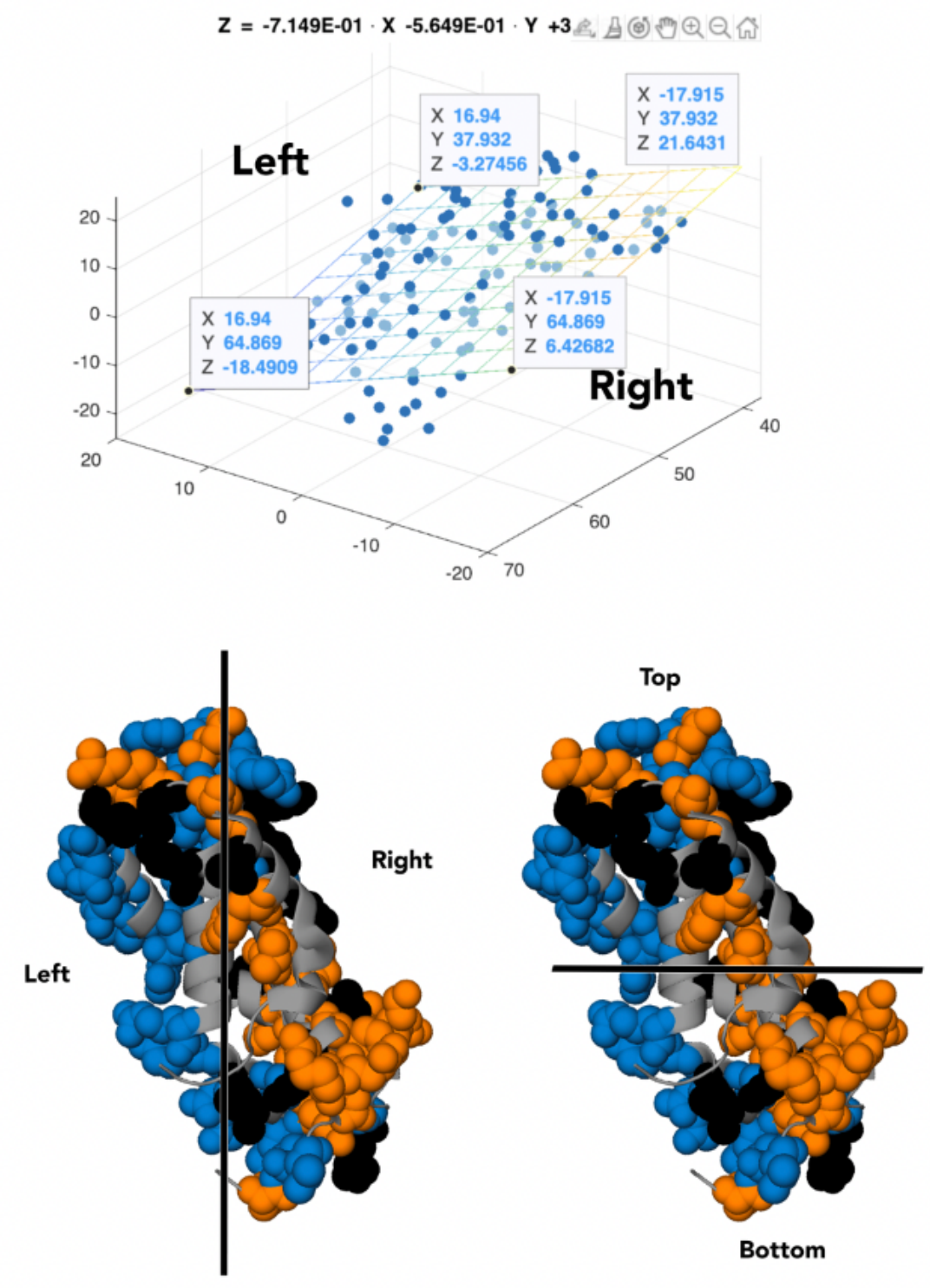

Supplementary Figure 2: Planes separating sp18 crystal structure. A.) Matlab was used to calculate the plane of best fit (Supplementary File 1) through the carbon alphas of the crystal structure (PDB 1GAK). B.) The plane of best fit divides the sp18 crystal structure into "left" and "right" sides. C.) A plane perpendicular to the plane of best fit divides the sp18 crystal structure into "top" and "bottom" sides. 


\section{Citations}

Aagaard, J. E., Springer, S. A., Soelberg, S. D., \& Swanson, W. J. (2013). Duplicate abalone egg coat proteins bind sperm lysin similarly, but evolve oppositely, consistent with molecular mimicry at fertilization. PLoS Genet, 9(2), e1003287. doi:10.1371/journal.pgen.1003287

Aagaard, J. E., Vacquier, V. D., MacCoss, M. J., \& Swanson, W. J. (2010). ZP domain proteins in the abalone egg coat include a paralog of VERL under positive selection that binds lysin and 18-kDa sperm proteins. Mol Biol Evol, 27(1), 193-203. doi:10.1093/molbev/msp221

Aagaard, J. E., Yi, X., MacCoss, M. J., \& Swanson, W. J. (2006). Rapidly evolving zona pellucida domain proteins are a major component of the vitelline envelope of abalone eggs. Proc Natl Acad Sci U S A, 103(46), 17302-17307. doi:10.1073/pnas.0603125103

Almagro Armenteros, J. J., Tsirigos, K. D., Sonderby, C. K., Petersen, T. N., Winther, O., Brunak, S., . . . Nielsen, H. (2019). SignalP 5.0 improves signal peptide predictions using deep neural networks. Nat Biotechnol, 37(4), 420-423. doi:10.1038/s41587-019-0036-z

Almeida, F. C., \& Desalle, R. (2009). Orthology, function and evolution of accessory gland proteins in the Drosophila repleta group. Genetics, 181(1), 235-245. doi:10.1534/genetics.108.096263

Anisimova, M., Bielawski, J. P., \& Yang, Z. (2001). Accuracy and power of the likelihood ratio test in detecting adaptive molecular evolution. Mol Biol Evol, 18(8), 1585-1592. doi:10.1093/oxfordjournals.molbev.a003945

Avella, M. A., Baibakov, B., \& Dean, J. (2014). A single domain of the ZP2 zona pellucida protein mediates gamete recognition in mice and humans. J Cell Biol, 205(6), 801-809. doi:10.1083/jcb.201404025

Bianchi, E., Doe, B., Goulding, D., \& Wright, G. J. (2014). Juno is the egg Izumo receptor and is essential for mammalian fertilization. Nature, 508(7497), 483-487. doi:10.1038/nature13203

Bianchi, E., \& Wright, G. J. (2020). Find and fuse: Unsolved mysteries in sperm-egg recognition. PLoS Biol, 18(11), e3000953. doi:10.1371/journal.pbio.3000953

Botwright, N. A., Zhao, M., Wang, T., McWilliam, S., Colgrave, M. L., Hlinka, O., ... Cummins, S. F. (2019). Greenlip Abalone (Haliotis laevigata) Genome and Protein Analysis Provides Insights into Maturation and Spawning. G3 (Bethesda), 9(10), 3067-3078. doi:10.1534/g3.119.400388

Cai, X., \& Clapham, D. E. (2008). Evolutionary genomics reveals lineage-specific gene loss and rapid evolution of a sperm-specific ion channel complex: CatSpers and CatSperbeta. PLoS One, 3(10), e3569. doi:10.1371/journal.pone.0003569

Carlisle, J. A., \& Swanson, W. J. (2020). Molecular mechanisms and evolution of fertilization proteins. J Exp Zool B Mol Dev Evol. doi:10.1002/jez.b.23004

Clark, N. L., Findlay, G. D., Yi, X., MacCoss, M. J., \& Swanson, W. J. (2007). Duplication and selection on abalone sperm lysin in an allopatric population. Mol Biol Evol, 24(9), 20812090. doi:10.1093/molbev/msm137

Clark, N. L., Gasper, J., Sekino, M., Springer, S. A., Aquadro, C. F., \& Swanson, W. J. (2009). Coevolution of interacting fertilization proteins. PLoS Genet, 5(7), e1000570. doi:10.1371/journal.pgen.1000570

Cooper, J. C., \& Phadnis, N. (2017). Parallel Evolution of Sperm Hyper-Activation Ca2+ Channels. Genome Biol Evol, 9(7), 1938-1949. doi:10.1093/gbe/evx131

Doty, K. A., Wilburn, D. B., Bowen, K. E., Feldhoff, P. W., \& Feldhoff, R. C. (2016). Co-option and evolution of non-olfactory proteinaceous pheromones in a terrestrial lungless salamander. J Proteomics, 135, 101-111. doi:10.1016/j.jprot.2015.09.019 
Ellerman, D. A., Pei, J., Gupta, S., Snell, W. J., Myles, D., \& Primakoff, P. (2009). Izumo is part of a multiprotein family whose members form large complexes on mammalian sperm. Mol Reprod Dev, 76(12), 1188-1199. doi:10.1002/mrd.21092

Findlay, G. D., Yi, X., Maccoss, M. J., \& Swanson, W. J. (2008). Proteomics reveals novel Drosophila seminal fluid proteins transferred at mating. PLoS Biol, 6(7), e178. doi:10.1371/journal.pbio.0060178

Galindo, B. E., Moy, G. W., Swanson, W. J., \& Vacquier, V. D. (2002). Full-length sequence of VERL, the egg vitelline envelope receptor for abalone sperm lysin. Gene, 288(1-2), 111117. doi:10.1016/s0378-1119(02)00459-6

Galindo, B. E., Vacquier, V. D., \& Swanson, W. J. (2003). Positive selection in the egg receptor for abalone sperm lysin. Proc Natl Acad Sci U S A, 100(8), 4639-4643. doi:10.1073/pnas.0830022100

Gan, H. M., Tan, M. H., Austin, C. M., Sherman, C. D. H., Wong, Y. T., Strugnell, J., . . Miller, A. D. (2019). Best Foot Forward: Nanopore Long Reads, Hybrid Meta-Assembly, and Haplotig Purging Optimizes the First Genome Assembly for the Southern Hemisphere Blacklip Abalone (Haliotis rubra). Front Genet, 10, 889. doi:10.3389/fgene.2019.00889

Gavrilets, S., \& Waxman, D. (2002). Sympatric speciation by sexual conflict. Proc Natl Acad Sci U S A, 99(16), 10533-10538. doi:10.1073/pnas.152011499

Grayson, P. (2015). Izumo1 and Juno: the evolutionary origins and coevolution of essential sperm-egg binding partners. $R$ Soc Open Sci, 2(12), 150296. doi:10.1098/rsos. 150296

Grayson, P., \& Civetta, A. (2012). Positive Selection and the Evolution of izumo Genes in Mammals. Int J Evol Biol, 2012, 958164. doi:10.1155/2012/958164

Huang, Y., Niu, B., Gao, Y., Fu, L., \& Li, W. (2010). CD-HIT Suite: a web server for clustering and comparing biological sequences. Bioinformatics, 26(5), 680-682. doi:10.1093/bioinformatics/btq003

Kamei, N., \& Glabe, C. G. (2003). The species-specific egg receptor for sea urchin sperm adhesion is EBR1, a novel ADAMTS protein. Genes Dev, 17(20), 2502-2507. doi:10.1101/gad.1133003

Killingbeck, E. E., \& Swanson, W. J. (2018). Egg Coat Proteins Across Metazoan Evolution. Curr Top Dev Biol, 130, 443-488. doi:10.1016/bs.ctdb.2018.03.005

Kozlov, A. M., Darriba, D., Flouri, T., Morel, B., \& Stamatakis, A. (2019). RAxML-NG: a fast, scalable and user-friendly tool for maximum likelihood phylogenetic inference. Bioinformatics, 35(21), 4453-4455. doi:10.1093/bioinformatics/btz305

Kresge, N., Vacquier, V. D., \& Stout, C. D. (2000). The high resolution crystal structure of green abalone sperm lysin: implications for species-specific binding of the egg receptor. $J \mathrm{Mol}$ Biol, 296(5), 1225-1234. doi:10.1006/jmbi.2000.3533

Kresge, N., Vacquier, V. D., \& Stout, C. D. (2001). The crystal structure of a fusagenic sperm protein reveals extreme surface properties. Biochemistry, 40(18), 5407-5413. doi: $10.1021 / \mathrm{bi002779v}$

Le, S. Q., \& Gascuel, O. (2008). An improved general amino acid replacement matrix. Mol Biol Evol, 25(7), 1307-1320. doi:10.1093/molbev/msn067

Lee, Y. H., Ota, T., \& Vacquier, V. D. (1995). Positive selection is a general phenomenon in the evolution of abalone sperm lysin. Mol Biol Evol, 12(2), 231-238. doi:10.1093/oxfordjournals.molbev.a040200

Lehmann, R. (2018). Matchmaking molecule for egg and sperm. Science, 361(6406), 974-975. doi:10.1126/science.aau8356

Lemoine, F., Domelevo Entfellner, J. B., Wilkinson, E., Correia, D., Davila Felipe, M., De Oliveira, T., \& Gascuel, O. (2018). Renewing Felsenstein's phylogenetic bootstrap in the era of big data. Nature, 556(7702), 452-456. doi:10.1038/s41586-018-0043-0 
Lewis, C. A., Leighton, D. L., \& Vacquier, V. D. (1980). Morphology of abalone spermatozoa before and after the acrosome reaction. $J$ Ultrastruct Res, 72(1), 39-46. doi:10.1016/s0022-5320(80)90133-1

Lyon, J. D., \& Vacquier, V. D. (1999). Interspecies chimeric sperm lysins identify regions mediating species-specific recognition of the abalone egg vitelline envelope. Dev Biol, 214(1), 151-159. doi:10.1006/dbio.1999.9411

MacDonald, R. J., Swift, G. H., Przybyla, A. E., \& Chirgwin, J. M. (1987). Isolation of RNA using guanidinium salts. Methods Enzymol, 152, 219-227. doi:10.1016/0076-6879(87)52023-7

Magel, R. C. W., S. H. (1997). Comparing the Powers of the Wald-Wolfowitz and KolmogorovSmirnov Tests. Biometrical Jornal, 39(6), 665.

Masonbrink, R. E., Purcell, C. M., Boles, S. E., Whitehead, A., Hyde, J. R., Seetharam, A. S., \& Severin, A. J. (2019). An Annotated Genome for Haliotis rufescens (Red Abalone) and Resequenced Green, Pink, Pinto, Black, and White Abalone Species. Genome Biol Evol, 11(2), 431-438. doi:10.1093/gbe/evz006

McGinnis, S., \& Madden, T. L. (2004). BLAST: at the core of a powerful and diverse set of sequence analysis tools. Nucleic Acids Res, 32(Web Server issue), W20-25. doi:10.1093/nar/gkh435

Metz, E. C., Robles-Sikisaka, R., \& Vacquier, V. D. (1998). Nonsynonymous substitution in abalone sperm fertilization genes exceeds substitution in introns and mitochondrial DNA. Proc Natl Acad Sci U S A, 95(18), 10676-10681. doi:10.1073/pnas.95.18.10676

Mozingo, N. M., Vacquier, V. D., \& Chandler, D. E. (1995). Structural features of the abalone egg extracellular matrix and its role in gamete interaction during fertilization. Mol Reprod Dev, 41(4), 493-502. doi:10.1002/mrd.1080410412

Nam, B. H., Kwak, W., Kim, Y. O., Kim, D. G., Kong, H. J., Kim, W. J., . . Kim, H. (2017). Genome sequence of pacific abalone (Haliotis discus hannai): the first draft genome in family Haliotidae. Gigascience, 6(5), 1-8. doi:10.1093/gigascience/gix014

Palmer, M. R., McDowall, M. H., Stewart, L., Ouaddi, A., MacCoss, M. J., \& Swanson, W. J. (2013). Mass spectrometry and next-generation sequencing reveal an abundant and rapidly evolving abalone sperm protein. Mol Reprod Dev, 80(6), 460-465. doi:10.1002/mrd.22182

Park, C. Y., Klammer, A. A., Kall, L., MacCoss, M. J., \& Noble, W. S. (2008). Rapid and accurate peptide identification from tandem mass spectra. J Proteome Res, 7(7), 30223027. doi:10.1021/pr800127y

Pei, J., Kim, B. H., \& Grishin, N. V. (2008). PROMALS3D: a tool for multiple protein sequence and structure alignments. Nucleic Acids Res, 36(7), 2295-2300. doi:10.1093/nar/gkn072

Pei, J., Tang, M., \& Grishin, N. V. (2008). PROMALS3D web server for accurate multiple protein sequence and structure alignments. Nucleic Acids Res, 36(Web Server issue), W30-34. doi:10.1093/nar/gkn322

Raj, I., Sadat Al Hosseini, H., Dioguardi, E., Nishimura, K., Han, L., Villa, A., . . Jovine, L. (2017). Structural Basis of Egg Coat-Sperm Recognition at Fertilization. Cell, 169(7), 1315-1326 e1317. doi:10.1016/j.cell.2017.05.033

Reams, A. B., \& Roth, J. R. (2015). Mechanisms of gene duplication and amplification. Cold Spring Harb Perspect Biol, 7(2), a016592. doi:10.1101/cshperspect.a016592

Sirot, L. K., Findlay, G. D., Sitnik, J. L., Frasheri, D., Avila, F. W., \& Wolfner, M. F. (2014). Molecular characterization and evolution of a gene family encoding both female- and male-specific reproductive proteins in Drosophila. Mol Biol Evol, 31(6), 1554-1567. doi:10.1093/molbev/msu114

Slater, G. S., \& Birney, E. (2005). Automated generation of heuristics for biological sequence comparison. BMC Bioinformatics, 6, 31. doi:10.1186/1471-2105-6-31 
Suyama, M., Torrents, D., \& Bork, P. (2006). PAL2NAL: robust conversion of protein sequence alignments into the corresponding codon alignments. Nucleic Acids Res, 34(Web Server issue), W609-612. doi:10.1093/nar/gkl315

Swanson, W. J., Nielsen, R., \& Yang, Q. (2003). Pervasive adaptive evolution in mammalian fertilization proteins. Mol Biol Evol, 20(1), 18-20. doi:10.1093/oxfordjournals.molbev.a004233

Swanson, W. J., \& Vacquier, V. D. (1995). Extraordinary divergence and positive Darwinian selection in a fusagenic protein coating the acrosomal process of abalone spermatozoa. Proc Natl Acad Sci U S A, 92(11), 4957-4961. doi:10.1073/pnas.92.11.4957

Swanson, W. J., \& Vacquier, V. D. (1997). The abalone egg vitelline envelope receptor for sperm lysin is a giant multivalent molecule. Proc Natl Acad Sci U S A, 94(13), 67246729. doi:10.1073/pnas.94.13.6724

Swanson, W. J., \& Vacquier, V. D. (2002). The rapid evolution of reproductive proteins. Nat Rev Genet, 3(2), 137-144. doi:10.1038/nrg733

Ulrich, A. S., Otter, M., Glabe, C. G., \& Hoekstra, D. (1998). Membrane fusion is induced by a distinct peptide sequence of the sea urchin fertilization protein bindin. $\mathrm{J}$ Biol Chem, 273(27), 16748-16755. doi:10.1074/jbc.273.27.16748

Vacquier, V. D., \& Moy, G. W. (1977). Isolation of bindin: the protein responsible for adhesion of sperm to sea urchin eggs. Proc Natl Acad Sci U S A, 74(6), 2456-2460. doi:10.1073/pnas.74.6.2456

Vacquier, V. D., \& Swanson, W. J. (2011). Selection in the rapid evolution of gamete recognition proteins in marine invertebrates. Cold Spring Harb Perspect Biol, 3(11), a002931. doi:10.1101/cshperspect.a002931

Wagstaff, B. J., \& Begun, D. J. (2005). Comparative genomics of accessory gland protein genes in Drosophila melanogaster and D. pseudoobscura. Mol Biol Evol, 22(4), 818-832. doi:10.1093/molbev/msi067

Wilburn, D. B., Arnold, J. A., Houck, L. D., Feldhoff, P. W., Feldhoff, R. C. (2017). Gene Duplication, Co-option, Structural Evolution, and Phenotypic Tango in the Courtship Pheromones of Plethodontid Salamanders. Herpetologica, 73, 206-219.

Wilburn, D. B., Tuttle, L. M., Klevit, R. E., \& Swanson, W. J. (2018). Solution structure of sperm lysin yields novel insights into molecular dynamics of rapid protein evolution. Proc Natl Acad Sci U S A, 115(6), 1310-1315. doi:10.1073/pnas.1709061115

Yang, Z. (2007). PAML 4: phylogenetic analysis by maximum likelihood. Mol Biol Evol, 24(8), 1586-1591. doi:10.1093/molbev/msm088

Zigler, K. S., McCartney, M. A., Levitan, D. R., \& Lessios, H. A. (2005). Sea urchin bindin divergence predicts gamete compatibility. Evolution, 59(11), 2399-2404. Retrieved from https://www.ncbi.nlm.nih.gov/pubmed/16396180 